The Free Internet Journal

for Organic Chemistry
Paper

Arkivoc 2017, part v, 141-147

\title{
A direct phosphine-mediated synthesis of polyfunctionalized 1-aminopyrroles from arylglyoxals, phenylhydrazine and acetylene diesters
}

\author{
Mahboobe Amirani Poorand, Mohammad Anary-Abbasinejad,* Ali Darehkordi \\ Department of chemistry, Vali-e-Asr University, Rafsanjan, 77176, Iran \\ Email: m.anary@vru.ac.ir
}

Received 10-25-2016

Accepted 06-14-2017

Published on line $08-23-2017$

\section{Abstract}

A new and efficient one-pot synthesis of 1-Aminopyrrole derivatives by three-component reaction of dialkyl acetylenedicarboxylates, phenylhydrazine and arylglyoxals in the presence of triphenylphosphine is described. The reactions were performed in dichloromethane at room temperature and neutral conditions and afforded good yields of products.

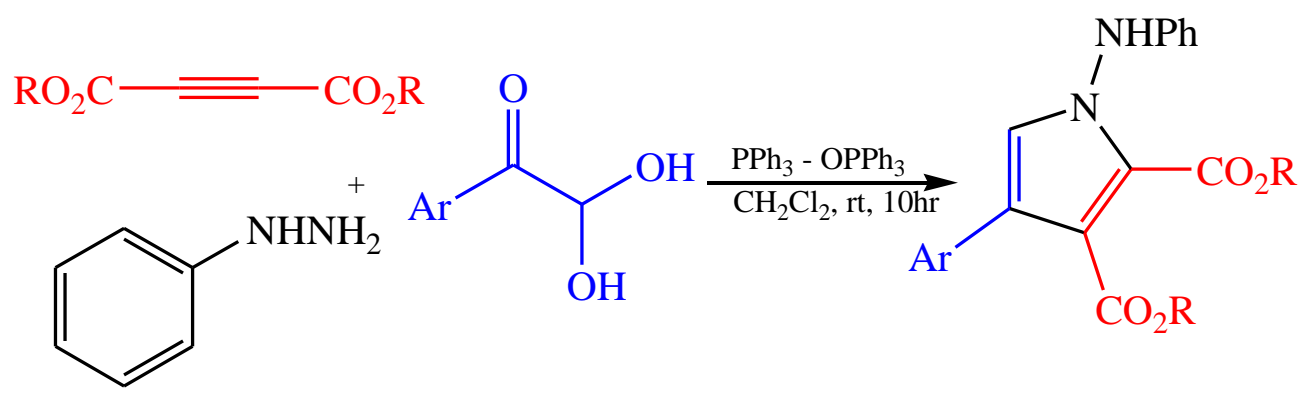

Ar: 4- $\mathrm{ClC}_{6} \mathrm{H}_{4}, 4-\mathrm{BrC}_{6} \mathrm{H}_{4}, 4-\mathrm{O}_{2} \mathrm{NC}_{6} \mathrm{H}_{4}, \mathrm{C}_{6} \mathrm{H}_{5}, 2-\mathrm{Naphthyl}, 4-\mathrm{MeC}_{6} \mathrm{H}_{4}$ R: $\mathrm{Me}, \mathrm{Et}, \mathrm{t}-\mathrm{Bu}$

12 examples

Yield: $70-87 \%$

Keywords: Phenylhydrazine, arylglyoxals, dialkyl acetylenedicarboxylates, 1-Aminopyrrole, triphenylphosphine 


\section{Introduction}

Pyrrole moieties are common subunits in numerous natural products ${ }^{1,2}$ and biological and medicinal important compounds ${ }^{3}$ and some are the building blocks for porphirine synthesis. ${ }^{3} 1$-aminopyrroles are important substructures as precursors for the synthesis of biologically active compounds..$^{4-7}$ Despite the wide application of 1-aminopyrroles, only a few methods are available for their preparation. ${ }^{8}$ Direct synthetic routs to these compounds are relative few and the reported methods suffer from severe reaction conditions, formation of by-products and tedious workup procedures. ${ }^{9-16}$

Multicomponent reactions (MCRs), especially three-component reactions, offer significant advantages over conventional linear-type syntheses because the combination of the reaction components to generate new products in a single step is easy and economic. ${ }^{17,18}$ Multi-component reactions of arylglyoxals have been recently attracted many attention for synthesis of a wide range of heterocyclic compounds. ${ }^{19-21}$ In continuation of our previous studies on the application of arrylglyoxals for the synthesis of heterocyclic compounds $\mathrm{s}^{22-25}$ here we wish to report a facile route to the synthesis of 1-aminopyrrole derivatives by a triphenylphosphine mediated three-component reaction between arylglyoxal derivatives, dialkyl acetylenedicarboxylates (DAADs) and phenylhydrazine (Scheme 1).

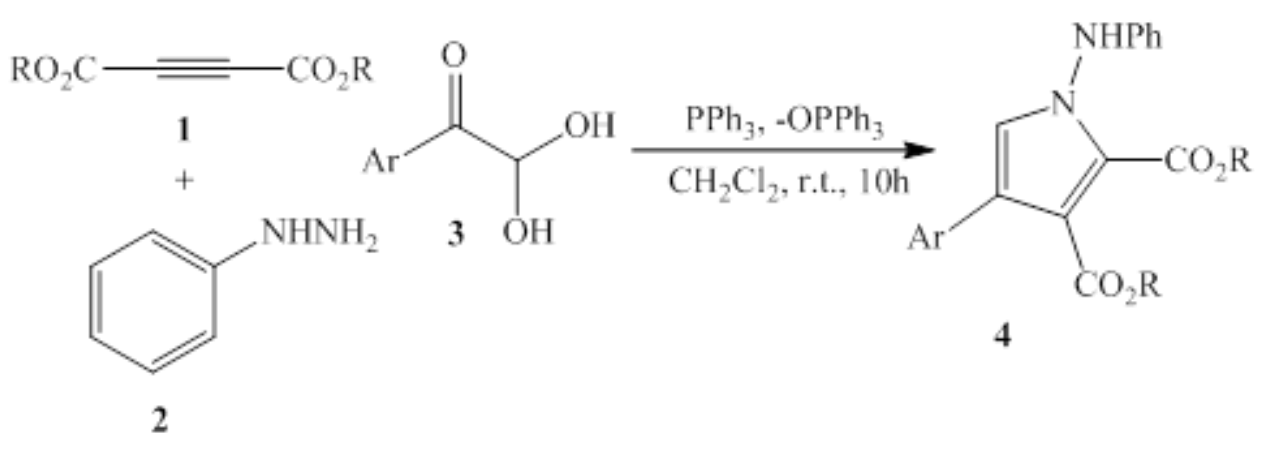

Scheme 1. Reaction between triphenylphosphine, arylglyoxals, phenylhydrazine and triphenylphosphine.

Table 1. The scope of the reaction between triphenylphosphine, arylglyoxals, phenylhydrazine and DAADs

\begin{tabular}{|c|c|c|c|}
\hline 4 & $R$ & $\mathrm{Ar}$ & Yield\% ${ }^{*}$ \\
\hline$a$ & $\mathrm{Me}$ & $4-\mathrm{ClC}_{6} \mathrm{H}_{4}$ & 87 \\
\hline b & $\mathrm{Me}$ & $4-\mathrm{BrC}_{6} \mathrm{H}_{4}$ & 83 \\
\hline c & $\mathrm{Me}$ & $4-\mathrm{O}_{2} \mathrm{NC}_{6} \mathrm{H}_{4}$ & 82 \\
\hline d & $\mathrm{Me}$ & $\mathrm{C}_{6} \mathrm{H}_{5}$ & 80 \\
\hline e & $t-\mathrm{Bu}$ & $4-\mathrm{BrC}_{6} \mathrm{H}_{4}$ & 75 \\
\hline f & $t$-Bu & $4-\mathrm{ClC}_{6} \mathrm{H}_{4}$ & 70 \\
\hline g & Et & 4- $\mathrm{BrC}_{6} \mathrm{H}_{4}$ & 86 \\
\hline $\mathrm{h}$ & Et & 4- $\mathrm{ClC}_{6} \mathrm{H}_{4}$ & 85 \\
\hline i & Et & 2-Naphthyl & 80 \\
\hline j & $t-\mathrm{Bu}$ & $4-\mathrm{MeC}_{6} \mathrm{H}_{4}$ & 79 \\
\hline k & $\mathrm{Me}$ & 4- $\mathrm{MeC}_{6} \mathrm{H}_{4}$ & 83 \\
\hline 1 & $\mathrm{Me}$ & 2-NaphC ${ }_{6} \mathrm{H}_{4}$ & 75 \\
\hline
\end{tabular}

*Isolated yields. Coditions: $\mathrm{CH}_{2} \mathrm{Cl}_{2}$, room temperature, $10 \mathrm{hs}$ 
Initially, to investigate the reaction between triphenylphosphine, DAADs, phenylhydrazine and arylglyoxals, dimethyl acetylenedicarboxylate (DMAD) was added to a mixture of phenylhydrazine and triphenyphosphine in $\mathrm{CH}_{2} \mathrm{Cl}_{2}$ as solvent at room temperature. Then 4-chlorophenylglyoxal monohydrate was added and the progress of the reaction was monitored by TLC. After $10 \mathrm{~h}$, the TLC of the mixture of the reaction showed only the presence of pyrrole derivative $4 a$ and triphenylphosphine oxide. Silica-gel chromatography afforded the product 1-Aminopyrrole $4 a$ in $87 \%$ yield. To investigate the scope of the reaction, diferrent DAADs were treated with triphenylphosphine, phenylhydrazine and different arylglyoxals and the corresponding 1 -aminopyrroles $\mathbf{4 b}$-i were obtained in good yields (Table 1 ).

\section{Results and Discussion}

The structures of compounds $4 \mathbf{a}-\mathbf{i}$ were deduced from their elemental analyses and their infrared (IR), ${ }^{1} \mathrm{H}$ $\mathrm{NMR}$, and ${ }^{13} \mathrm{CNMR}$ spectral data. $500-\mathrm{MH}_{\mathrm{Z}}{ }^{1} \mathrm{H}$ NMR spectrum of $4 \mathrm{a}$ exhibited three sharp signals at $\delta 3.75$, 3.85 , and $6.60 \mathrm{ppm}$ for two methoxy groups protons and the proton of pyrrole ring, respectively. Aromatic protons resonated between 7.00 and $7.38 \mathrm{ppm}$. The $\mathrm{NH}$ proton resonated at $7.80 \mathrm{ppm}$ as a broad signal. The ${ }^{13} \mathrm{C}$ NMR spectrum of compound 4 a showed 15 distinct resonances in agreement with the proposed structure. The structural assignments made on the basis of the NMR spectra of compound $\mathbf{4 a}$ were supported by its IR spectrum. The ester carbonyl groups exhibited strong absorption bands at about 1731 and $1713 \mathrm{~cm}^{-1}$. Finally, the structure of $4 a$ was unambiguously confirmed by its X-Ray crystal structure (Figure 1).

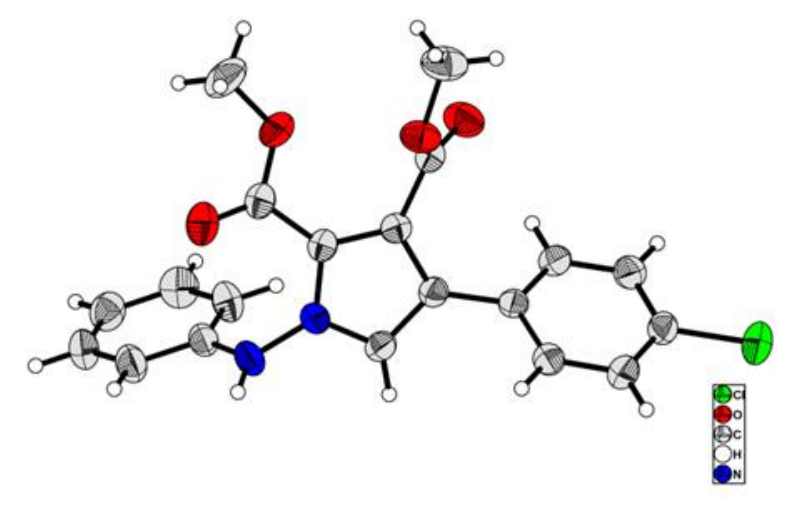

Figure 1. ORTEP diagram of $4 a$ with atom numbering scheme. Thermal ellipsoids are shown at $50 \%$ probability (CCDC number 1508905).

The suggested mechanism for formation of 1-aminopyrroles by the reaction between triphenylphosphine, arylglyoxal derivatives, DAADs and phenylhydrazine is showed in Scheme 2. Three component reaction between triphenylphosphine, DAAD and phenylhydrazine afforded phosphorane 5. The addition of intermediate $\mathbf{5}$ to arylgyoxal derivative lead to intermediate $\mathbf{6}$ which converted to the 1-amininopyrrle derivative by an intramolecular Wittig reaction and elimination of a molecule of water.

In conclusion, here we report a three-component reaction between dialkyl acetylenedicarboxylates, phenylhydrazine and arylglyoxals promoted by triphenylphosphine to produce functionalized 1-aminopyrrole derivatives in good yields. The reaction performed under neutral conditions, and the substances can be mixed without any activation or modification to afford high yields of products. 


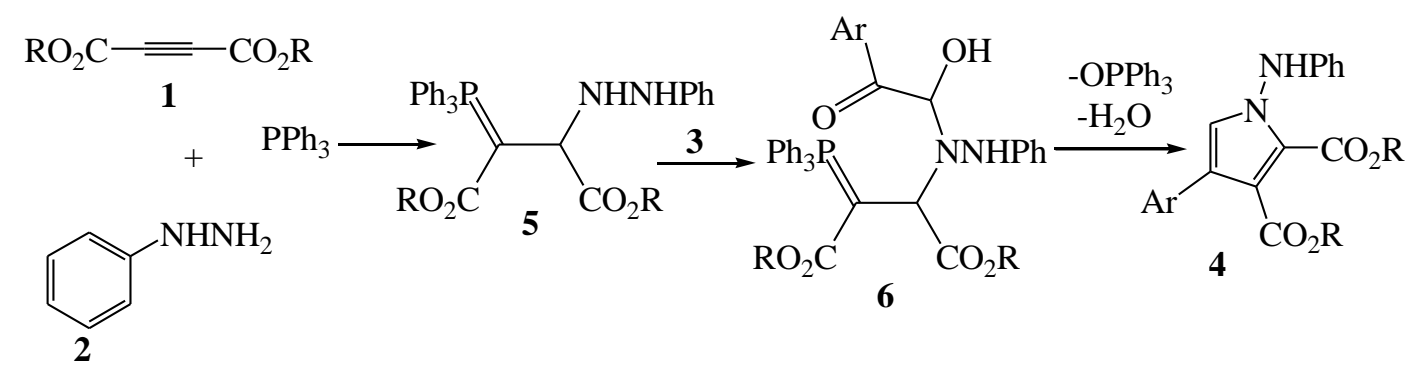

Scheme 2. Suggested mechanism for formation of 1-aminopyrrole derivatives 4a-i.

\section{Experimental Section}

General. All the utilized arylglyoxals were prepared by the $\mathrm{SeO}_{2}$-oxidation of the related aryl methylketones on the basis of the reported procedure and used as their monohydrates.1 Elemental analyses were performed using a Heraeus CHN-O-Rapidanalyzer. IR spectra were recorded on a Shimadzu IR-470 spectrometer. ${ }^{26} \mathrm{H}$, and ${ }^{13} \mathrm{C}$ NMRspectra were recorded on Bruker DRX-400 Avance spectrometer at 400 and $100 \mathrm{MH}_{\mathrm{z}}$, respectively. The chemicals used in this work purchased from Merck and were used without further purification.

General procedure of dimethyl 5-(4-chlorophenyl)-1-(phenylamino)-1H-pyrrole-2,3-dicarboxylate (4a). A mixture of dimethyl acetylenedicarboxylate $(142 \mathrm{mg}, 1 \mathrm{mmol})$ in $\mathrm{CH}_{2} \mathrm{Cl}_{2}(3 \mathrm{~mL})$ was added drop wise to a magnetically stirred solution of triphenylphosphine $(262 \mathrm{mg}, 1 \mathrm{mmol})$ and phenylhydrazine $(108 \mathrm{mg}, 1 \mathrm{mmol})$ in $\mathrm{CH}_{2} \mathrm{Cl}_{2}(10 \mathrm{~mL})$. The reaction mixture was then stirred for $1 \mathrm{~min}$. 4-Chlorophenylglyoxal monohydrate (186 $\mathrm{mg}, 1 \mathrm{mmol}$ ) was added, and the reaction mixture was stirred for more $10 \mathrm{hr}$ at room temperature. The solvent was evaporated, and the residue was purified by column chromatography on silica gel using ethyl acetate-hexane mixture ( $4: 1$ ratio) as eluent to give the product $(308 \mathrm{mg}, 80 \%)$ as a white solid.

Dimethyl 5-(4-chlorophenyl)-1-(phenylamino)-1H-pyrrole-2,3-dicarboxylate (4a). Yield: (308 mg, 80\%); white solid; mp 134-136 ${ }^{\circ} \mathrm{C}$. IR (KBr) (v̄max, cm-1): $3333(\mathrm{NH}), 1731,1713(\mathrm{C}=0) .{ }^{1} \mathrm{H} \mathrm{NMR}\left(\mathrm{CDCl}_{3}, 400 \mathrm{MHz}\right): \delta 3.85(3$ $\mathrm{H}, \mathrm{s}), 3.75(3 \mathrm{H}, \mathrm{s}), 6.60\left(2 \mathrm{H}, \mathrm{t}^{3} \mathrm{~J}_{\mathrm{HH}} 8 \mathrm{HZ}\right), 7.00\left(1 \mathrm{H}, \mathrm{t},{ }^{3} \mathrm{~J}_{\mathrm{HH}} 8 \mathrm{~Hz}_{\mathrm{Z}}\right), 7.19(1 \mathrm{H}, \mathrm{S}, \mathrm{Pyr}-\mathrm{H}), 7.26\left(2 \mathrm{H}, \mathrm{t},{ }^{3} \mathrm{~J}_{\mathrm{HH}} 8 \mathrm{~Hz}\right), 7.38$ $(4 \mathrm{H}, \mathrm{m}), 7.80(1 \mathrm{H}, \mathrm{s}, \mathrm{NH} \mathrm{PhNH}) .{ }^{13} \mathrm{C} \mathrm{NMR}\left(\mathrm{CDCl}_{3}, 100 \mathrm{MHz}_{\mathrm{z}}\right): \delta$ 51.6, 52.0 (aliphathic carbon), 113.0, 119.0, 120.0, 120.1, 122.1, 125.5, 126.3, 126.4, 128.3, 128.4, 130.8, 132.7, 147.2 (aromatic carbons), 165.5, 169.6 (C=O). Calcd. for $\left(\mathrm{C}_{20} \mathrm{H}_{17} \mathrm{ClN}_{2} \mathrm{O}_{4}\right): \mathrm{C}, 62.42 ; \mathrm{H}, 4.45 ; \mathrm{N}, 7.28 \%$. Found: $\mathrm{C}, 62.33 ; \mathrm{H}, 4.52 ; \mathrm{N}, 7.35 \%$.

Dimethyl 5-(4-bromophenyl)-1-(phenylamino)-1H-pyrrole-2,3-dicarboxylate (4b). Yield: (356 mg, 83\%); white solid; mp $150-153^{\circ} \mathrm{C}$. IR ( $\left.\mathrm{KBr}\right)\left(\overline{\mathrm{v}}_{\mathrm{max}}, \mathrm{cm}^{-1}\right): 3335(\mathrm{NH}), 1715(\mathrm{C}=\mathrm{O}) .{ }^{1} \mathrm{H} \mathrm{NMR}\left(\mathrm{CDCl}_{3}, 400 \mathrm{MHz}\right): \delta 3.75(3 \mathrm{H}, \mathrm{s})$, $3.84(3 \mathrm{H}, \mathrm{s}), 6.60\left(2 \mathrm{H}, \mathrm{d},{ }^{3} \mathrm{~J}_{\mathrm{HH}} 8 \mathrm{~Hz}\right), 7.00\left(1 \mathrm{H}, \mathrm{t}^{3} \mathrm{~J}_{\mathrm{HH}} 8 \mathrm{~Hz}\right), 7.19(1 \mathrm{H}, \mathrm{S}, \mathrm{Pyr}-\mathrm{H}), 7.26\left(1 \mathrm{H}, \mathrm{t},{ }^{3} J_{\mathrm{HH}} 8 \mathrm{~Hz}\right), 7.33(2 \mathrm{H}$, $\left.\mathrm{d},{ }^{3} \mathrm{~J}_{\mathrm{HH}} 8 \mathrm{~Hz}\right), 7.52\left(2 \mathrm{H}, \mathrm{d}, \mathrm{t}{ }^{3} \mathrm{JHH}_{\mathrm{HH}} 8 \mathrm{~Hz}\right), 7.79(1 \mathrm{H}, \mathrm{s}, \mathrm{NH} \mathrm{PhNH}) .{ }^{13} \mathrm{C} \mathrm{NMR}\left(\mathrm{CDCl}_{3}, 100 \mathrm{MH}_{\mathrm{Z}}\right): \delta 52.1,52.5$ (aliphathic carbon), 113.7, 120.7, 121.3, 122.6, 123.1, 125.9, 127.5, 128.8, 129.2, 129.4, 131.1, 147.1 (aromatic carbons), 160.1, 166.1 ( $\mathrm{C}=\mathrm{O})$. Calcd. for $\left(\mathrm{C}_{20} \mathrm{H}_{17} \mathrm{BrN}_{2} \mathrm{O}_{4}\right)$ : C, 55.96; $\mathrm{H}, 3.99 ; \mathrm{N}, 6.53 \%$. Found: $\mathrm{C}, 55.85 ; \mathrm{H}, 4.01 ; \mathrm{N}, 6.60 \%$.

Dimethyl 5-(4-nitrophenyl)-1-(phenylamino)-1H-pyrrole-2,3-dicarboxylate (4c). Yield: (323 mg, 82\%); white solid; mp $165-167^{\circ} \mathrm{C}$. IR (KBr) ( $\left.\bar{v}_{\max }, \mathrm{cm}^{-1}\right)$ : $3321(\mathrm{NH}), 1717(\mathrm{C}=\mathrm{O})$.

${ }^{1} \mathrm{H} N M R\left(\mathrm{CDCl}_{3}, 400 \mathrm{MHz}\right): \delta 3.77(3 \mathrm{H}, \mathrm{s}), 3.87(3 \mathrm{H}, \mathrm{s}), 6.61\left(2 \mathrm{H}, \mathrm{d},{ }^{3} \mathrm{~J}_{\mathrm{HH}} 8 \mathrm{~Hz}\right), 7.01\left(1 \mathrm{H}, \mathrm{t}^{3} \mathrm{~J}_{\mathrm{HH}} 8 \mathrm{~Hz}\right), 7.25(1 \mathrm{H}$, S, Pyr-H), $7.28\left(1 \mathrm{H}, \mathrm{t},{ }^{3} \mathrm{~J}_{\mathrm{HH}} 8 \mathrm{~Hz}\right), 7.61\left(2 \mathrm{H}, \mathrm{d},{ }^{3} J_{\mathrm{HH}} 8 \mathrm{~Hz}\right), 7.81(1 \mathrm{H}, \mathrm{s}, \mathrm{NH} \mathrm{PhNH}), 8.26\left(2 \mathrm{H}, \mathrm{d}, \mathrm{t}^{3} J_{\mathrm{HH}} 8 \mathrm{~Hz}\right) .{ }^{13} \mathrm{C}$ $\mathrm{NMR}\left(\mathrm{CDCl}_{3}, 100 \mathrm{MH}_{\mathrm{z}}\right): \delta$ 52.2, 52.6 (aliphathic carbon), 113.8, 119.6, 120.5, 121.0, 122.8, 124.9, 126.6, 128.1, 
129.5, 139.6, 146.7, 147.4 (aromatic carbons), 159.9, $165.6(\mathrm{C}=\mathrm{O})$. Calcd. for $\left(\mathrm{C}_{20} \mathrm{H}_{17} \mathrm{~N}_{3} \mathrm{O}_{6}\right): \mathrm{C}, 60.76 ; \mathrm{H}, 4.33 ; \mathrm{N}$, $10.63 \%$. Found: C, 60.83; H, 4.21; N, 10.52\%

Dimethyl 4-phenyl-1-(phenylamino)-1H-pyrrole-2,3-dicarboxylate (4d). Yield: (304 mg, 87\%); white solid; mp $153-155^{\circ} \mathrm{C}$. IR (KBr) ( $\left.\bar{v}_{\max }, \mathrm{cm}-1\right): 3263(\mathrm{NH}), 1716(\mathrm{C}=0) .{ }^{1} \mathrm{H} \mathrm{NMR}\left(\mathrm{CDCl}_{3}, 400 \mathrm{MHz}\right): \delta 3.76(3 \mathrm{H}, \mathrm{s}), 3.85(3 \mathrm{H}, \mathrm{s})$, $6.61\left(2 \mathrm{H}, \mathrm{t} \mathrm{t}^{3} \mathrm{JHH}_{\mathrm{HH}} 8 \mathrm{~Hz}\right), 6.99\left(1 \mathrm{H}, \mathrm{t}^{3} \mathrm{~J}_{\mathrm{HH}} 8 \mathrm{H}_{\mathrm{Z}}\right), 7.28(1 \mathrm{H}, \mathrm{S}, \mathrm{Pyr}-\mathrm{H}), 7.31\left(1 \mathrm{H}, \mathrm{t}^{3} \mathrm{~J}_{\mathrm{HH}} 8 \mathrm{H}_{\mathrm{Z}}\right), 7.41\left(2 \mathrm{H}, \mathrm{t},{ }^{3} \mathrm{~J}_{\mathrm{HH}} 8 \mathrm{H}_{\mathrm{Z}}\right)$,

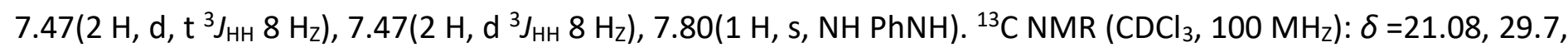
52.0, 52.5 (aliphathic carbon), 113.7, 119.7, 120.8, 121.7, 122.5, 123.1, 126.1, 127.2, 127.5, 128.9, 129.4, 132.8, 147.9 (aromatic carbons), 160.1, $166.3(\mathrm{C}=\mathrm{O})$. Calcd. for $\left(\mathrm{C}_{20} \mathrm{H}_{18} \mathrm{~N}_{2} \mathrm{O}_{4}\right)$ : C, 68.56; $\mathrm{H}, 5.18 ; \mathrm{N}, 8.00 \%$. Found: C, 68.64; H, 5.06; N, 8.19\%.

Di-t-butyl 5-(4-bromophenyl)-1-(phenylamino)-1H-pyrrole-2,3-dicarboxylate (4e). Yield: (309 mg, 75\%); White solid; $\mathrm{mp} 90-93^{\circ} \mathrm{C}$. IR $(\mathrm{KBr})\left(\bar{v}_{\max }, \mathrm{cm}^{-1}\right): 3380(\mathrm{NH}), 1720,1699(\mathrm{C}=\mathrm{O}) .{ }^{1} \mathrm{H} \mathrm{NMR}\left(\mathrm{CDCl}_{3}, 400 \mathrm{MHz}\right): \delta 1.39$ $(9 \mathrm{H}, \mathrm{s}), 1.45(9 \mathrm{H}, \mathrm{s}), 6.67\left(2 \mathrm{H}, \mathrm{d},{ }^{3} \mathrm{~J}_{\mathrm{HH}} 8 \mathrm{~Hz}\right), 6.98\left(1 \mathrm{H}, \mathrm{t}^{3} \mathrm{~J}_{\mathrm{HH}} 8 \mathrm{~Hz}\right), 7.25\left(2 \mathrm{H}, \mathrm{t},{ }^{3} \mathrm{JHH}_{\mathrm{HH}} 8 \mathrm{H}_{\mathrm{Z}}\right), 7.28(1 \mathrm{H}, \mathrm{S}, \mathrm{Pyr}-\mathrm{H})$, $7.32\left(2 \mathrm{H}, \mathrm{d},{ }^{3} \mathrm{JHH}_{\mathrm{HH}} 8 \mathrm{~Hz}\right), 7.51\left(2 \mathrm{H}, \mathrm{t},{ }^{3} \mathrm{~J}_{\mathrm{HH}} 8 \mathrm{~Hz}\right), 7.70\left(1 \mathrm{H}, \mathrm{s}, \mathrm{NH} \mathrm{PhNH}, \mathrm{t}^{3} \mathrm{JHH}_{\mathrm{HH}} 8 \mathrm{H}_{\mathrm{Z}}\right) .{ }^{13} \mathrm{C} \mathrm{NMR}\left(\mathrm{CDCl}_{3}, 100 \mathrm{MH}_{\mathrm{Z}}\right): \delta=$ 27.4, 81.2, 82.1 (aliphathic carbon), 131.3, 113.3, 120.3, 120.4, 120.6, 121.9, 124.0, 128.8, 129.6, 130.7, 147.9 (aromatic carbons), 158.7, $163.3(\mathrm{C}=\mathrm{O})$. Calcd. for $\left(\mathrm{C}_{26} \mathrm{H}_{29} \mathrm{BrN}_{2} \mathrm{O}_{4}\right)$ : C, 60.82; $\mathrm{H}, 5.69 ; \mathrm{N}, 5.46 \%$. Found: $\mathrm{C}, 60.71$; $H, 5.81 ; N, 5.58 \%$.

Di-t-butyl 5-(4-chlorophenyl)-1-(phenylamino)-1H-pyrrole-2,3-dicarboxylate (4f). Yield: (257 mg, 70\%); White

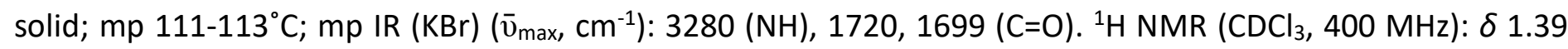
$(9 \mathrm{H}, \mathrm{s}),, 1.45(9 \mathrm{H}, \mathrm{s}), 6.59\left(2 \mathrm{H}, \mathrm{d},{ }^{3} \mathrm{~J}_{\mathrm{HH}} 8 \mathrm{~Hz}\right), 6.59\left(2 \mathrm{H}, \mathrm{d},{ }^{3} J_{\mathrm{HH}} 8 \mathrm{H}_{\mathrm{Z}}\right), 6.98\left(1 \mathrm{H}, \mathrm{t},{ }^{3} J_{\mathrm{HH}} 8 \mathrm{~Hz}\right), 7.25\left(1 \mathrm{H}, \mathrm{d},{ }^{3} J_{\mathrm{HH}} 8\right.$ $\left.\mathrm{H}_{\mathrm{z}}\right), 7.28(1 \mathrm{H}, \mathrm{s}, \mathrm{Pyr}-\mathrm{H}), 7.36\left(3 \mathrm{H}, \mathrm{d}, \mathrm{t}{ }^{3} \mathrm{~J}_{\mathrm{HH}} 8 \mathrm{~Hz}\right), 7.70(1 \mathrm{H}, \mathrm{s}, \mathrm{NH} \mathrm{PhNH}) .{ }^{13} \mathrm{C} \mathrm{NMR}\left(\mathrm{CDCl}_{3}, 100 \mathrm{MHZ}\right): \delta 27.4$, 81.2, 82.1 (aliphathic carbon), 131.3, 113.3, 120.3, 120.4, 120.6, 121.9, 124.0, 128.8, 129.6, $130.7,147.9$ (aromatic carbons), 158.7, 163.3 (C=O). Calcd. for $\left(\mathrm{C}_{26} \mathrm{H}_{29} \mathrm{ClN}_{2} \mathrm{O}_{4}\right)$ : C, 60.82; $\mathrm{H}, 5.69 ; \mathrm{N}, 5.46 \%$. Found: $\mathrm{C}, 60.94$; $\mathrm{H}, 5.53 ; \mathrm{N}, 5.32 \%$.

Diethyl 5-(4-bromophenyl)-1-(phenylamino)-1H-pyrrole-2,3-dicarboxylate (4g). Yield: (393 mg, 86\%); white solid; mp $153-155^{\circ} \mathrm{C}$. IR $(\mathrm{KBr})\left(\bar{v}_{\max }, \mathrm{cm}^{-1}\right): 3328(\mathrm{NH}), 1709(\mathrm{C}=\mathrm{O}) .{ }^{1} \mathrm{H} \mathrm{NMR}\left(\mathrm{CDCl}_{3}, 400 \mathrm{MHz}\right): \delta 1.21\left(3 \mathrm{H}, \mathrm{t}, 3^{3} \mathrm{JH}_{\mathrm{H}}\right.$ $\left.8 \mathrm{H}_{\mathrm{Z}}\right), 1.31\left(3 \mathrm{H}, \mathrm{t}, 3^{3} \mathrm{JHH}_{\mathrm{H}} 8 \mathrm{H}_{\mathrm{Z}}\right), 4.22\left(2 \mathrm{H}, \mathrm{q},{ }^{3} \mathrm{~J}_{\mathrm{HH}} 8 \mathrm{H}_{\mathrm{Z}}\right), 4.32\left(2 \mathrm{H}, \mathrm{q},{ }^{3} \mathrm{~J}_{\mathrm{HH}} 8 \mathrm{H}_{\mathrm{Z}}\right), 6.62\left(2 \mathrm{H}, \mathrm{d},{ }^{3} \mathrm{~J}_{\mathrm{HH}} 8 \mathrm{~Hz}, \operatorname{arom}\right), 7.00(1$ $\mathrm{H}, \mathrm{t}, 3^{3} \mathrm{HH}_{\mathrm{H}} 8 \mathrm{~Hz}$, arom), $\left.7.18(1 \mathrm{H}, \mathrm{S}, \mathrm{Pyr}-\mathrm{H})\right), 7.27\left(1 \mathrm{H}, \mathrm{t},{ }^{3} \mathrm{~J}_{\mathrm{HH}} 8 \mathrm{~Hz}, \operatorname{arom}\right), 7.39(4 \mathrm{H}, \mathrm{m}, \operatorname{arom}), 7.81(1 \mathrm{H}, \mathrm{s}, \mathrm{NH}$ $\mathrm{PhNH}) .{ }^{13} \mathrm{C} \mathrm{NMR}\left(\mathrm{CDCl}_{3}, 100 \mathrm{MH}_{\mathrm{z}}\right): \delta$ 13.9, 14.0, 61.1, 61.6 (aliphathic carbon), 113.8, 120.2, 120.5, 120.8, 121.2, 122.6, 125.7, 129.3, 129.4, 131.96, 147.8 (aromatic carbons), 159.7, 165.6 (C=O). Calcd. for $\left(\mathrm{C}_{22} \mathrm{H}_{21} \mathrm{BrN}_{2} \mathrm{O}_{4}\right): \mathrm{C}, 57.78 ; \mathrm{H}, 4.63 ; \mathrm{N}, 6.13 \%$. Found: $\mathrm{C}, 57.62 ; \mathrm{H}, 4.77 ; \mathrm{N}, 6.26 \%$.

Diethyl 5-(4-chlorophenyl)-1-(phenylamino)-1H-pyrrole-2,3-dicarboxylate (4h). Yield: (351 ng, 85\%); white solid; $\mathrm{mp} 153-155^{\circ} \mathrm{C}$. IR ( $\left.\mathrm{KBr}\right)\left(\overline{\mathrm{v}}_{\mathrm{max}}, \mathrm{cm}^{-1}\right): 3328(\mathrm{NH}), 1709(\mathrm{C}=\mathrm{O})$.

${ }^{1} \mathrm{H}$ NMR $\left(\mathrm{CDCl}_{3}, 400 \mathrm{MHz}\right): \delta 1.23\left(3 \mathrm{H}, \mathrm{t},{ }^{3} \mathrm{~J}_{\mathrm{HH}} 8 \mathrm{~Hz}\right), 1.31\left(3 \mathrm{H}, \mathrm{t},{ }^{3} J_{\mathrm{HH}} 8 \mathrm{~Hz}\right), 4.22\left(2 \mathrm{H}, \mathrm{q}^{3} J_{\mathrm{HH}} 8 \mathrm{~Hz}\right), 4.32(2 \mathrm{H}, \mathrm{q}$ $\left.{ }^{3} J_{\mathrm{HH}} 8 \mathrm{~Hz}\right), 6.62\left(2 \mathrm{H}, \mathrm{d},{ }^{3} \mathrm{~J}_{\mathrm{HH}} 8 \mathrm{~Hz}\right.$, arom), $7.00\left(1 \mathrm{H}, \mathrm{t},{ }^{3} J_{\mathrm{HH}} 8 \mathrm{~Hz}\right.$, arom), $\left.7.18(1 \mathrm{H}, \mathrm{S}, \mathrm{Pyr}-\mathrm{H})\right), 7.27\left(1 \mathrm{H}, \mathrm{t},{ }^{3} \mathrm{JHH}_{\mathrm{H}} 8 \mathrm{~Hz}\right.$,

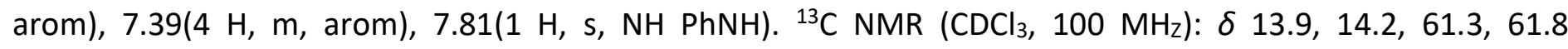
(aliphathic carbon), 114.0, 120.2, 120.5, 120.9, 121.2, 122.6, 126.1, 129.7, 129.9, 131.96, 147.8 (aromatic carbons), 159.7, 165.6 (C=O). Calcd. for $\left(\mathrm{C}_{22} \mathrm{H}_{21} \mathrm{ClN}_{2} \mathrm{O}_{4}\right): \mathrm{C}, 57.78 ; \mathrm{H}, 4.63 ; \mathrm{N}, 6.13 \%$. Found: $\mathrm{C}, 57.67 ; \mathrm{H}, 4.76 ; \mathrm{N}$, $6.27 \%$.

Diethyl 4-(naphthalen-2-yl)-1-(phenylamino)-1H-pyrrole-2,3-dicarboxylate (4i). Yield: (342 mg, 80\%); white solid; mp 97-99 ${ }^{\circ} \mathrm{C}$. IR ( $\left.\mathrm{KBr}\right)\left(\bar{v}_{\max }, \mathrm{cm}^{-1}\right): 3304(\mathrm{NH}), 1720(\mathrm{C}=\mathrm{O}) .{ }^{1} \mathrm{H} \mathrm{NMR}\left(\mathrm{CDCl}_{3}, 400 \mathrm{MHz}\right): \delta 1.25\left(3 \mathrm{H}, \mathrm{t},{ }^{3} \mathrm{~J}_{\mathrm{HH}} 8\right.$ $\left.\mathrm{Hz}_{\mathrm{z}}\right), 1.31\left(3 \mathrm{H}, \mathrm{t},{ }^{3} \mathrm{~J}_{\mathrm{HH}} 8 \mathrm{~Hz}\right), 4.24\left(2 \mathrm{H}, \mathrm{q}^{3} \mathrm{~J}_{\mathrm{HH}} 8 \mathrm{~Hz}\right), 4.35\left(2 \mathrm{H}, \mathrm{q}^{3} \mathrm{~J}_{\mathrm{HH}} 8 \mathrm{~Hz}\right), 6.67\left(2 \mathrm{H}, \mathrm{d},{ }^{3} J_{\mathrm{HH}} 8 \mathrm{~Hz}, \operatorname{arom}\right), 7.01(1 \mathrm{H}$, t, ${ }^{3} J_{\mathrm{HH}} 8 \mathrm{~Hz}$, arom), $7.29\left(2 \mathrm{H}, \mathrm{t},{ }^{3} \mathrm{~J}_{\mathrm{HH}} 8 \mathrm{~Hz}\right.$, arom) ), 7.48-7.54(2 H, m), 7.60-7.63(1 H, m, arom), 7.85-7.89 (3H, m, naph, py), 7.95(1 H, s, NH PhNH). ${ }^{13} \mathrm{C} \mathrm{NMR}\left(\mathrm{CDCl}_{3}, 100 \mathrm{MH}_{\mathrm{z}}\right): \delta 13.9,14.1,61.0$ (aliphathic carbon), 113.8, 
$119.9,121.3,121.5,125.9,126.0,126.1,126.3,127.6,127.9,128.2,129.4,130.4,132.4,133.5,148.0$ (aromatic carbons), 165.9(C=O). Calcd. for $\left(\mathrm{C}_{26} \mathrm{H}_{24} \mathrm{~N}_{2} \mathrm{O}_{4}\right): \mathrm{C}, 72.88 ; \mathrm{H}, 5.65 ; \mathrm{N}, 6.54 \%$.Found: $\mathrm{C}, 73.02 ; \mathrm{H}, 5.51 ; \mathrm{N}, 6.43 \%$

Di-t-butyl 1-(phenylamino)-4-(p-tolyl)-1H-pyrrole-2,3-dicarboxylate (4j). Yield: (260 mg, 75\%); White solid; $\mathrm{mp} 142-144^{\circ} \mathrm{C}$. IR (KBr) ( $\left.\bar{v}_{\max }, \mathrm{cm}^{-1}\right): 3320(\mathrm{NH}), 1725,1705(\mathrm{C}=0)$.

${ }^{1} \mathrm{H}$ NMR $\left(\mathrm{CDCl}_{3}, 400 \mathrm{MHz}\right): \delta 1.40(9 \mathrm{H}, \mathrm{s}), 1.45(9 \mathrm{H}, \mathrm{s}), 2.40\left(1 \mathrm{H}, \mathrm{s}, \mathrm{CH}_{3}\right), 6.61\left(2 \mathrm{H}, \mathrm{d}^{3} \mathrm{~J}_{\mathrm{HH}} 8 \mathrm{~Hz}_{\mathrm{z}}\right), 6.98\left(1 \mathrm{H}, \mathrm{t}^{3} \mathrm{JHH}_{\mathrm{H}}\right.$ $8 \mathrm{~Hz}), 7.02(1 \mathrm{H}, \mathrm{S}, \mathrm{Pyr}-\mathrm{H}), 7.20\left(2 \mathrm{H}, \mathrm{d},{ }^{3} \mathrm{~J}_{\mathrm{HH}} 8 \mathrm{~Hz}\right), 7.26(2 \mathrm{H}, \mathrm{m}), 7.35\left(2 \mathrm{H}, \mathrm{d},{ }^{3} \mathrm{~J}_{\mathrm{HH}} 8 \mathrm{~Hz}\right), 7.73(1 \mathrm{H}, \mathrm{s}, \mathrm{NH} \mathrm{PhNH}) .{ }^{13} \mathrm{C}$ $\mathrm{NMR}\left(\mathrm{CDCl}_{3}, 100 \mathrm{MH}_{\mathrm{z}}\right): \delta 27.4$ (aliphathic carbon), 81.2, 82.1, 131.3, 113.3, 120.3, 120.4, 120.6, 121.9, 124.0, 128.8, 129.6, 130.7, 147.9 (aromatic carbons), 159.4, 164.2 (C=O). Calcd. for $\left(\mathrm{C}_{27} \mathrm{H}_{32} \mathrm{~N}_{2} \mathrm{O}_{4}\right): \mathrm{C}, 72.30 ; \mathrm{H}, 7.19 ; \mathrm{N}$, 6.25;\%. Found: C, 72.15; H, 7.33; N, 6.38;\%.

Dimethyl 1-(phenylamino)-4-(p-tolyl)-1H-pyrrole-2,3-dicarboxylate (4k). Yield: (316 mg, 83\%); white solid; mp $105^{\circ} \mathrm{C}$. IR ( $\left.\mathrm{KBr}\right)\left(\bar{v}_{\max }, \mathrm{cm}^{-1}\right): 3310(\mathrm{NH}), 1717(\mathrm{C}=0) .{ }^{1} \mathrm{H}$ NMR $\left(\mathrm{CDCl}_{3}, 400 \mathrm{MHz}\right): \delta 2.40(3 \mathrm{H}, \mathrm{s}), 3.67(3 \mathrm{H}, \mathrm{s}), 3.88$ $(3 \mathrm{H}, \mathrm{s}), 6.62\left(2 \mathrm{H}, \mathrm{d},{ }^{3} \mathrm{~J}_{\mathrm{HH}} 8 \mathrm{~Hz}\right), 7.00\left(1 \mathrm{H}, \mathrm{t} \mathrm{t}^{3} \mathrm{~J}_{\mathrm{HH}} 8 \mathrm{~Hz}\right), 7.20(1 \mathrm{H}, \mathrm{S}, \mathrm{Pyr}-\mathrm{H}), 7.21-7.24(2 \mathrm{H}, \mathrm{m}), 7.26-7.28(2 \mathrm{H}, \mathrm{m})$, $7.35\left(2 \mathrm{H}, \mathrm{d}, \mathrm{t}^{3} \mathrm{~J}_{\mathrm{HH}} 8 \mathrm{~Hz}\right), 7.81(1 \mathrm{H}, \mathrm{s}, \mathrm{NH} \mathrm{PhNH}) .{ }^{13} \mathrm{C} \mathrm{NMR}\left(\mathrm{CDCl}_{3}, 100 \mathrm{MH}_{\mathrm{Z}}\right): \delta 21.2,52.0,52.5$ (aliphathic carbon), 113.7, 119.5, 120.7, 121.7, 122.5, 126.0, 127.4, 129.4, 129.8, 137.0, 147.9 (aromatic carbons), 160.2, 166.4(C=O). Calcd. for $\left(\mathrm{C}_{21} \mathrm{H}_{20} \mathrm{~N}_{2} \mathrm{O}_{4}\right): \mathrm{C}, 69.22 ; \mathrm{H}, 5.53 ; \mathrm{N}, 7.69 \%$. Found: $\mathrm{C}, 69.37 ; \mathrm{H}, 5.40 ; \mathrm{N}, 7.56 \%$.

Dimethyl 4-(naphthalen-2-yl)-1-(phenylamino)-1H-pyrrole-2,3-dicarboxylate (4I) Yield: (300 mg, 75\%); white solid; mp $119^{\circ} \mathrm{C}$. IR ( $\left.\mathrm{KBr}\right)\left(\overline{\mathrm{v}}_{\max }, \mathrm{cm}^{-1}\right)$ : $3286(\mathrm{NH}), 1723(\mathrm{C}=0) .{ }^{1} \mathrm{H} \mathrm{NMR}\left(\mathrm{CDCl}_{3}, 400 \mathrm{MHz}\right): \delta 3.79(3 \mathrm{H}, \mathrm{s}), 3.87(3$ $\mathrm{H}, \mathrm{s}), 3.88,6.66\left(2 \mathrm{H}, \mathrm{d},{ }^{3} \mathrm{~J}_{\mathrm{HH}} 8 \mathrm{~Hz}\right), 7.00\left(1 \mathrm{H}, \mathrm{t}^{3} \mathrm{~J}_{\mathrm{HH}} 8 \mathrm{~Hz}\right), 7.29\left(3 \mathrm{H}, \mathrm{t}^{3} \mathrm{~J}_{\mathrm{HH}} 8 \mathrm{~Hz}_{\mathrm{Z}}\right), 7.48-7.55(2 \mathrm{H}, \mathrm{m}), 7.56-7.61(1$ $\mathrm{H}, \mathrm{m})$, 7.86-7.90 (4 H, m, arom, Pyr-H)), $7.94(1 \mathrm{H}, \mathrm{s}, \mathrm{NH} \mathrm{PhNH}) .{ }^{13} \mathrm{C} \mathrm{NMR}\left(\mathrm{CDCl}_{3}, 100 \mathrm{MH}_{\mathrm{z}}\right): \delta 52.1,52.5$ (aliphathic carbon), 113.8, 119.8, 121.0, 121.7, 122.6, 125.9, 126.0, 126.3, 126.4, 127.6, 128.0, 128.3, 129.4, $130.3,132.5,133.5,147.9$ (aromatic carbons), 160.2, 166.4(C=O). Calcd. for $\left(\mathrm{C}_{24} \mathrm{H}_{20} \mathrm{~N}_{2} \mathrm{O}_{4}\right)$ : C, 71.99; $\mathrm{H}, 5.03 ; \mathrm{N}$, 7.00\%. Found: C, 71.85; H, 5.17; N, 7.13\%.

\section{Supplementary Material}

The experimental procedures and IR, ${ }^{1} \mathrm{H}$ NMR and ${ }^{13} \mathrm{C}$ NMR spectra associated with this article are available as supplementary data.

\section{References}

1. Herbert, R. B. In The Alkaloids; Grundon, M. F. Ed. Chemical Society: London, 1982, 12.

2. 2. Falk, H. The Chemistry of Linear Oligopyrroles and Bile Pigments, Springer-Verlag, 1989; p 47. https://doi.org/10.1007/978-3-7091-6938-4

3. Gribble, G. W. In Comprehensive Heterocyclic Chemistry II, Katritzky, A. R.; Rees, C. W.; Scriven, E. F. V. Eds. Pergamon: Oxford, 1996; Vol. 2.

4. Fuhrhop, J. H.; Smith, K. M. In Porphyrins and Metalloporphyrins, Smith, K. M., Ed. Elsevier: New York, 1975; pp 20-21.

5. Franck, B.; Nonn, A. Angew. Chem. Int. Ed. Engl. 1995, 34, 1795.

https://doi.org/10.1002/anie.199517951

6. Sessler, J. L; Weghorn, S. J. In Expanded, Contracted \& Isomeric Porphyrins; Pergamon Press: Oxford, 1997, p 15. 
7. Effland, R. C.; Klein, J. T. U.S. Patent 4,546,105, 1985. Chem.Abstr. 1986, 104, 186307.

8. Kulagowski, J.; Janusz, J.; Leeson, P. D. UK. Patent 2,265,372, 1993. Chem. Abstr.1993, 120, 134504.

9. Sommer, S. Angew. Chem.Int. Ed. 1979, 18, 695. https://doi.org/10.1002/anie.197906951

10. Bean, G. P. In The Chemistry of Heterocyclic Compounds, Pyrroles; Jones, R. A., Ed.; John Wiley \& Sons: New York, 1990, Vol. 48.

11. Cirrincione, G.; Almerico, A. M.; Aiello, E.;Dattolo, G. In The Chemistry of Heterocyclic Compounds, Pyrroles; Jones, R. A. Ed. John Wiley \& Sons: New York, 1992, Vol. 48.

12. Seino, H.; Ishii, Y.; Sasagawa, T.; Hidai, M. J. Am. Chem. Soc. 1995, 117, 12181. https://doi.org/10.1021/ja00154a019

13. McLeod, M.; Boudreault, N.;Leblanc, Y. J. Org. Chem. 1996, 61, 1180. https://doi.org/10.1021/jo9518260

14. Arcadi, A.; Anacardio,R.; D'Anniballe, G.; Gentile, M. Synlett 1997, 1315. https://doi.org/10.1055/s-1997-1006

15. Attanasi, O. A.; De Crescentini, L.;Favi, G.; Filippone, P.; Mantellini, F.; Santeusanio, S. J. Org. Chem. 2002, $67,8178$.

https://doi.org/10.1021/jo025656k

16. Attanasi, O. A.; Favi, G.; Filippone, P.; Golobic, A.; Stanovnik, B.; Svete, J. J. Org.Chem. 2005, $70,4307$. https://doi.org/10.1021/jo0480343

17. Yavari, I.; Bayat, M, J.; Sirouspour, M.; Souri, S. Tetrahedron 2010, 66, 7995. https://doi.org/10.1016/j.tet.2010.08.016

18. Shengule,S, R.; Karuso, P. Australian J. Chem. 2011, 64, 1617. https://doi.org/10.1071/CH11358

19. Jiang, B.; Zhang, Q. Y. Li.;Pindi, S. J. Tu.; Li, G. Org. Lett. 2012, 14, 700. https://doi.org/10.1021/ol203166c

20. Jiang, B.; Yi, M. S.; Shi, F.; Tu, S. J.;Pindi, S.; McDowell, P.; Li, G. Chem. Commun. 2012, $48,808$. https://doi.org/10.1039/C1CC15913E

21. Eftekhari-Sis, B.; Zirak, M.; Akbari, A. Chem. Rev. 2013, 113, 2958. https://doi.org/10.1021/cr300176g

22. Anary-Abbasinejad, M; Talebizadeh, M. J. Iran. Chem. Soc. 2014, 11, 963. https://doi.org/10.1007/s13738-013-0362-x

23. Masoudi, M.; Anary-Abbasinejad, M. J. Chem. Res. 2015, 145.

24. Anary-Abbasinejad, M.; Falahati, J. Iran. Chem. Soc. 2015, 12, 1415. https://doi.org/10.1007/s13738-015-0608-x

25. Masoudi, M.; Anary-Abbasinejad, M. Tetrahedron Lett. 2016, 57, 103. https://doi.org/10.1016/i.tetlet.2015.11.075

26. Riley, H. A.; Gray, A. R. Organic Syntheses 1943, 2, 509. https://doi.org/10.1007/s13738-013-0362-x 